\title{
Topics In Pediatrics
}

\section{Chiropractic Treatments for Idiopathic Scoliosis: A Narrative Review Based on SOSORT Outcome Criteria}

\author{
Mark W. Momingstar, DC, PhD, ${ }^{\text {a }}$ Clayton J. Stitzel, DC, ${ }^{\text {b }}$ Aatif Siddiqui, DC, ${ }^{c}$ and Brian Dovorany, DC ${ }^{\text {d }}$
}

\begin{abstract}
Objective: The purpose of this review was to evaluate the current body of literature on chiropractic treatment of idiopathic scoliosis against the 2014 consensus paper of the Society on Scoliosis Orthopedic and Rehabilitation Treatment (SOSORT) and the Scoliosis Research Society (SRS) Non-Operative Management Committee for outcome reporting in nonoperative treatments.

Methods: A search of the PubMed and Index to Chiropractic Literature databases for studies published from January 2000 through February 2016 detailing specific treatments and outcomes for idiopathic scoliosis was conducted.

Results: A total of 27 studies that discussed chiropractic scoliosis treatments were identified. Of these, there were 15 case reports, 10 case series, 1 prospective cohort, and 1 randomized clinical trial. Of the 27 studies, only 2 described their outcomes as recommended in the 2014 SOSORT and SRS Non-Operative Management Committee consensus paper.

Conclusion: The 2014 SOSORT and SRS Non-Operative Committee consensus paper details the format and types of outcomes they collectively believe are the most important and relevant to the patient. Among the chiropractic studies located in this review, 2 described outcomes consistent with how SOSORT recommends they be reported. Given that these consensus papers form the basis for nonoperative treatment recommendations and outcome reporting, future chiropractic studies should seek to report their outcomes as recommended by these papers. This will allow for better interprofessional collaboration and methodologic comparison. (J Chiropr Med 2017;16:64-71)
\end{abstract}

Key Indexing Terms: Chiropractic; Rehabilitation; Scoliosis; Spine

\section{INTRODUCTION}

Idiopathic scoliosis is a condition with a long history of proposed nonoperative treatments. These treatments have varied widely, but often with the common goal of stabilizing or correcting the Cobb angle and/or providing pain relief. ${ }^{1,2}$

Chiropractic medicine first began in the United States as a healing profession in $1895 .{ }^{3}$ Chiropractic medicine was

\footnotetext{
${ }^{\mathrm{a}}$ Natural Wellness \& Pain Relief Center, Grand Blanc, MI.

${ }^{\mathrm{b}}$ Lancaster Spinal Health Center, Lititz, PA.

${ }^{\mathrm{c}}$ Esprit Wellness Center, New York, NY.

${ }^{\mathrm{d}}$ Posture \& Spine Care Center, Green Bay, WI.

Corresponding author: Mark W. Morningstar, DC, PhD, 8293

Office Park Drive, Grand Blanc, MI 48439. Tel.: +1-810-

814-0422. (e-mail: drmorningstar@nwprc.com).

Paper submitted March 30, 2016; in revised form October 7, 2016; accepted October 12, 2016.

1556-3707

(C) 2016 National University of Health Sciences.

http://dx.doi.org/10.1016/j.jcm.2016.10.004
}

originally created under the premise that spinal misalignments, called vertebral subluxations, could adversely affect nearby peripheral nerves and result in downstream organic health disorders. ${ }^{4}$ Despite chiropractic medicine's declared focus on the spine and conditions thereof, comparatively little research has been performed on the relationship between chiropractic care and idiopathic scoliosis. Because of the variety of treatment methods employed by doctors of chiropractic, ${ }^{5}$ it is difficult to compare the effectiveness of various methods against one another. For example, chiropractic physicians, within their respective scopes of practice, may use spinal manipulation, nutrition, exercises, traction, bracing, or electric stimulation as scoliosis treatment therapies. Although these therapies are common to other health professions as well, it is possible that the results differ when performed by chiropractic physicians. They may also be reported differently than by other health professions. The focus of this article is less on the treatment results specifically, but rather on the manner in which they are reported. Thus, the goal of this review was to identify 
peer-reviewed chiropractic literature specifically regarding idiopathic scoliosis and compare the reporting of results to that recommended by the 2014 SOSORT and SRS consensus paper ${ }^{6}$ and Weiss. ${ }^{7}$ This review may provide insight into how future chiropractic studies can use this consensus paper to strengthen outcome reporting and foster interprofessional communication.

\section{METHODS}

We searched the PubMed and Index to Chiropractic Literature databases for studies published from January 2000 through February 2016 detailing specific treatments and outcomes for idiopathic scoliosis. We searched using the key words chiropractic AND scoliosis. We avoided other search terms, such as manipulation, bracing, and exercises, because other disciplines also perform these same physiotherapeutic procedures. We wanted to focus only on those studies wherein the procedures administered were either performed or directly supervised by chiropractic physicians. Each of the chiropractic studies we identified was compared with the individual outcome criteria identified. ${ }^{6,7}$ A table was created for each outcome criterion that totaled the number of chiropractic studies identified that satisfied each given criterion. We did not search further back as it is increasingly difficult to obtain full-text articles prior to the year 2000 in electronic format. Additionally, because SOSORT was organized in 2004, studies much earlier than 2000 would not, in all likelihood, report their outcomes in a manner consistent with SOSORT recommendations.

\section{RESULTS}

The 2 databases were cross-referenced (78 citations in PubMed and 221 citations in ICL). For this review, the following inclusion criteria were used $^{1}$ : full-text studies available, ${ }^{2}$ at least one outcome measure reported, ${ }^{3}$ study available in English, ${ }^{4}$ and no abstracts, redundancies, or studies not reporting specific treatment methods and outcomes. We were left with a total of 27 studies for evaluation that were published between January 2000 and February 2016. These studies were broken down into the following study designs: 15 total case reports, 10 case series, 1 prospective cohort study, and 1 randomized clinical trial. When subcategorizing these studies based on the type of treatment used, we found that 5 studies used chiropractic manipulation only, 15 studies used manipulation plus exercises, 2 studies used exercises only, 3 studies used bracing only (as prescribed and fitted by a chiropractor), and 2 studies used chiropractic manipulation and rigid bracing (orthotist-fitted). According to the 2014 SOSORT and SRS consensus paper, ${ }^{6}$ as well as Weiss, ${ }^{7}$ specific outcome data were specifically recommended. In summary, here are the main criteria they outlined, which formed the basis for this review ${ }^{6,7}$ :

1. Initial patient age and Risser stage (baseline should be Risser $0-2$ )

2. Initial Cobb angle (ie, Cobb between $10^{\circ}$ and $30^{\circ}$, $>30^{\circ}$, and $>50^{\circ}$ )

3. Cobb angle at skeletal maturity (Risser 5)

4. Percentage of patients whose Cobb angle improved by $\geq 6^{\circ}$

5. Percentage of patient whose Cobb angle stabilized within $\pm 5^{\circ}$

6. Percentage of patients whose Cobb angle progressed by $\geq 6^{\circ}$

7. Percentage of patients whose curves progressed beyond $50^{\circ}$

8. Skeletally immature patients managed through end of growth

9. Skeletally mature patients managed for at least 5 years.

The initial 2005 SOSORT consensus paper, ${ }^{8}$ which preceded the 2014 SOSORT Consensus paper, formed the framework for the 2006 Council on Chiropractic Guidelines and Practice Parameters Guidelines for the management of idiopathic scoliosis. ${ }^{9}$ Therefore, it was appropriate to review the various chiropractic studies relating to scoliosis treatment in relation to these above criteria.

\section{Manipulation Only}

A total of 6 studies were identified. Table 1 compares these studies against the recommended outcome criteria. Jaszewski and Sorbara reported on the treatment of a 7-year-old patient with juvenile idiopathic scoliosis. ${ }^{10}$ She received manipulation using the Pierce technique on 4 office visits in 1 month, after which a follow-up scoliosis radiograph was taken. It revealed a curve reduction of $13^{\circ}$ to $5^{\circ}$. This case report does indicate a curve correction of at least $6^{\circ}$. However, this patient was only followed for 1 month. Although the authors note that the patient continued treatment for additional symptoms unrelated to the scoliosis, additional Cobb angle measurements are not reported at later follow-ups. Another case report by Khauv and Dickholtz outlined the treatment of a 15-year-old with a $44^{\circ}$ scoliosis that decreased to $32^{\circ}$ after 5 months of upper cervical manipulation (National Upper Cervical Chiropractic Association [NUCCA]). ${ }^{11}$ Although the patient was managed for a total of $>4$ years, a long-term Cobb angle measurement is not reported. Chung and Salminen reported on a case of a 10 -year-old girl with a $35^{\circ}$ thoracolumbar scoliosis. ${ }^{12}$ She was treated using NUCCA manipulation over a total of 25 weeks. Follow-up radiograph at 25 weeks revealed a Cobb angle reduction of $10^{\circ}$. The authors do not report a baseline Risser stage, nor do they provide a long-term follow-up measurement at skeletal maturity. Chen and Chiu reported the case of another 15-year-old girl 
Table I. Comparison of Studies Against the Recommended Outcome Criteria

\begin{tabular}{|c|c|c|c|c|c|c|c|c|c|}
\hline Authors & $\begin{array}{l}\text { Risser } \\
0-2\end{array}$ & $\begin{array}{l}\text { Initial } \\
\text { Cobb }\end{array}$ & $\begin{array}{l}\text { Cobb at } \\
\text { Risser } 5\end{array}$ & $\begin{array}{l}\%>6^{\circ} \\
\text { Corr }\end{array}$ & $\% \pm 5^{\circ}$ & $\begin{array}{l}\%>6^{\circ} \\
\text { Prog }\end{array}$ & $\begin{array}{l}\text { Prog } \\
>50^{\circ}\end{array}$ & $\begin{array}{l}\text { Tx Risser } \\
0-4\end{array}$ & $\begin{array}{l}\text { Tx Risser } 5 \\
\text { for } 5 \mathrm{y}\end{array}$ \\
\hline \multicolumn{10}{|l|}{ Manipulation Only } \\
\hline Jaszewski and Sorbara $^{10}$ & NR & Yes & NR & NA & Yes & NA & NA & NR & NA \\
\hline Khauv and Dickholtz ${ }^{11}$ & NR & Yes & NR & NA & Yes & NA & & NR & NA \\
\hline Chung and Salminen ${ }^{12}$ & NR & Yes & NR & NA & Yes & NA & NA & NR & NA \\
\hline Chen and $\mathrm{Chiu}^{13}$ & NR & Yes & NR & NA & Yes & NA & NA & No & NA \\
\hline Chen and Lantz ${ }^{14}$ & NR & Yes & NR & No & No & No & No & NR & NA \\
\hline \multicolumn{10}{|l|}{ Chiropractic Bracing } \\
\hline Waldrop et $\mathrm{al}^{15}$ & Yes & Yes & NR & Yes & NA & NA & NA & NR & NA \\
\hline Morningstar $^{16}$ & NR & Yes & NR & Yes & Yes & Yes & NR & NR & NR \\
\hline Morningstar et $\mathrm{al}^{17}$ & NR & Yes & NR & Yes & Yes & Yes & NR & NR & NR \\
\hline \multicolumn{10}{|l|}{ Exercises Only } \\
\hline Golembiewski and Catanzaro $^{18}$ & NR & Yes & NR & NR & NR & NR & NR & NR & NR \\
\hline Lewis et $\mathrm{al}^{19}$ & NA & Yes & NA & NR & Yes & NR & NR & NR & NR \\
\hline \multicolumn{10}{|l|}{ Manipulation + Bracing } \\
\hline Rowe et $\mathrm{al}^{20}$ & Yes & Yes & NR & NR & NR & NR & NR & NR & NA \\
\hline Morningstar $^{21}$ & Yes & Yes & NR & NR & NR & NR & NR & NR & NA \\
\hline \multicolumn{10}{|l|}{ Manipulation + Traction } \\
\hline Stitzel et $\mathrm{al}^{23}$ & NR & Yes & NR & NR & NR & NR & NR & NR & NA \\
\hline \multicolumn{10}{|l|}{ Manipulation + Exercise } \\
\hline Fedorchuk et $\mathrm{al}^{24}$ & NR & Yes & NR & NR & NR & NR & NR & NR & NA \\
\hline Nektalov $^{25}$ & NA & Yes & NA & NA & NA & NA & NA & NA & NR \\
\hline Jones $^{26}$ & NR & Yes & NR & NA & NA & NA & NA & NR & NA \\
\hline Ray et $\mathrm{al}^{27}$ & NA & Yes & NA & NA & NA & NA & NA & NA & NR \\
\hline Bowler and Conlen ${ }^{29}$ & Yes & Yes & NR & NA & NA & NA & NA & NR & NA \\
\hline Blum $^{31}$ & NA & Yes & NA & NA & NA & NA & NA & NA & NR \\
\hline Morningstar et $\mathrm{al}^{32}$ & NR & Yes & NR & NA & NA & NA & NA & NR & NR \\
\hline Morningstar and Joy ${ }^{33}$ & NA & Yes & NA & NA & NA & NA & NA & NA & NR \\
\hline Morningstar and Strauchman ${ }^{34}$ & NA & Yes & NA & NA & NA & NA & NA & NA & NR \\
\hline Morningstar et $\mathrm{al}^{35,36}$ & NA & Yes & NA & NA & NA & NA & NA & NA & NR \\
\hline Morningstar $^{37}$ & NA & Yes & NA & NA & NA & NA & NA & NA & NR \\
\hline Morningstar $^{38}$ & No & Yes & Yes & NA & NA & NA & NA & No & NR \\
\hline Dovorany et $\mathrm{al}^{39}$ & NR & Yes & Yes & NA & NA & NA & NA & Yes & NR \\
\hline Morningstar et $\mathrm{al}^{40}$ & NR & Yes & NR & NA & NA & NA & NA & No & NR \\
\hline Villafane et $\mathrm{al}^{41}$ & NR & Yes & NR & NA & NA & NA & NA & NR & NR \\
\hline
\end{tabular}

$\% \pm 5^{\circ}$, percentage of patients whose Cobb angle stabilized within $\pm 5^{\circ} ; \%>6^{\circ}$ Corr, percentage of patients whose Cobb angle improved by $\geq 6^{\circ} ; \%>6^{\circ}$ Prog, percentage of patients whose Cobb angle progressed by $\geq 6^{\circ}$; Cobb at Risser 5, Cobb angle at skeletal maturity; Initial Cobb, initial Cobb angle (ie, Cobb between $10^{\circ}$ and $30^{\circ},>30^{\circ}$, and $>50^{\circ}$ ); $N A$, not applicable to study; $N R$, not reported in study; $\operatorname{Prog}>50^{\circ}$, percentage of patients whose curves progressed $>50^{\circ}$; Risser 0-2, Risser stage (baseline should be Risser 0-2); Tx Risser 0-4, skeletally immature patients managed through end of growth; Tx Risser 5 for $5 y$, skeletally mature patients managed for at least 5 years.

with a history of progressive scoliosis whose curve progressed despite a 4-year history of combined physical therapy and bracing treatment with the Milwaukee brace. ${ }^{13}$ The patient was treated for 18 months using full spine manipulation, after which a follow-up radiograph revealed a curve correction of $16^{\circ}$. Risser staging is not reported, and it is unknown if the 18-month follow-up radiograph was obtained at skeletal maturity.

A prospective cohort study involving 42 patients was reported by Chen and Lantz. ${ }^{14}$ Using full spine manipulation and postural ergonomic training plus heel lifts, they found no change in curve measurements after an average 14-month follow-up. The authors did not provide a breakdown of the entire cohort into curve severity or Risser staging. Therefore, it was difficult to determine, based on the data presented, if this intervention helped to stabilize the majority of curves, or if the treatment was ineffective as the authors concluded.

The key deficit in the manipulation-only subgroup was that none of the studies attempted to follow the patient through skeletal maturity. Although Khauv and Dickholtz followed the patient for 4 years, the patient initiated treatment at age 15 , and was likely near the end of skeletal growth if not already there. ${ }^{11}$ Therefore, the authors' results would have been significantly strengthened if they had followed her for an additional year. The remainder of the studies did not report outcomes consistent with SOSORT criteria other than Cobb angle changes. 


\section{Bracing Only (as Performed by Chiropractors)}

This category of treatment entails the use of some type of orthosis specifically fitted by a chiropractor. In our literature search, we found 3 such studies. One of these studies was a case report by Waldrop et al describing the use of a SpineCor brace in treating a 10-year-old girl for idiopathic scoliosis. ${ }^{15}$ Follow-up at 8 months revealed complete resolution of the scoliosis, and a 1-year follow-up indicated maintenance of the correction. The outcomes for this group are summarized in Table 1.

The 2 other studies were published by Morningstar et al. ${ }^{16,17}$ Both of these were case series studies in adult patients with a history of idiopathic scoliosis. In the first case series, Morningstar identified 62 patients who wore a scoliosis activity suit for 1 year and reviewed their pain and radiographic findings. ${ }^{16}$ In this study, the entire cohort was divided into 2 groups: one group with Risser stage $<5$ and the other $>5$. In the immature group $(<5), 16$ of the 26 patients had curve correction $>6^{\circ}, 6$ of the 26 remained within $5^{\circ}$ of baseline, and 4 patients' curves increased $>6^{\circ}$. Adult patients had corrections in 14 of 36 cases; 20 were unchanged, and 2 progressed. These outcomes were observed after 1 year of wearing the suit.

In a second follow-up study, Morningstar et al evaluated the changes recorded in adult patients wearing the scoliosis activity suit for 18 months. ${ }^{17}$ Of the entire 53-patient cohort, 79\% achieved correction, 17\% were unchanged, and $4 \%$ progressed.

Although the results of all 3 studies are noteworthy, they do not conform to the SOSORT criteria described herein. Waldrop et al did not follow their patient through skeletal maturity. ${ }^{15}$ Morningstar et al did not follow skeletally immature patients through Risser 5, nor did they follow adult patients for the recommended 5 years. ${ }^{16,17}$

\section{Exercises Only}

Two of the studies we found dealt with exercises only for scoliosis treatment. Golembiewski and Catanzaro evaluated the ability of an isometric hip flexor exercise to reduce a lumbar scoliosis in an adult patient. ${ }^{18}$ No outcomes were reported in the study beyond a stress radiograph taken while the patient was performing the exercise. Therefore, it is impossible to know if the exercise could provide a lasting correction.

The second study evaluated an isometric exercise station called the ATM2 and its ability to influence scoliosis. This study was a case report reporting on a 55-year-old female patient who underwent treatment with the ATM2 for adhesive capsulitis and calcific tendinitis. ${ }^{19}$ Although postural photography revealed improved postural characteristics, the before and after radiographic measurements of the scoliosis were identical. Table 1 summarizes these studies.

\section{Manipulation Plus Bracing}

A couple of studies we retrieved reported the results of treatment consisting of spinal manipulation plus rigid bracing. One of the studies, by Rowe et al, was a randomized controlled clinical trial involving 6 patients. ${ }^{20}$ This study was methodologically the strongest of any study we retrieved. The patients were randomized into different groups receiving different combinations of therapy. Of the 6 patients, 1 achieved success after 6 months of chiropractic manipulation and rigid bracing. Although the study design was strong, they did not report on the outcomes of these patients at or after skeletal maturity. However, they did report the baseline Risser staging for each patient. Interestingly, 4 of the 6 patients had a baseline Risser of 3 or 4 , and yet still progressed despite management. The lone patient who improved began with a Risser 1. Importantly, the goal of this study was not to determine effectiveness of treatment, but rather to investigate the plausibility of the study design and implementation.

In a small case series, Morningstar reported on 4 patients with idiopathic scoliosis who participated in a rehabilitation program while concurrently wearing a rigid brace as well. ${ }^{21}$ After 3 months of participating in the combined approach, the average $\mathrm{Cobb}$ angle reduction was $13.5^{\circ}$. Of note was that 3 of the 4 patients had baseline curvatures that were greater than the usual standard of care $\left(>60^{\circ}\right.$ and $\left.>80^{\circ}\right)$, yet still achieved an average correction $>6^{\circ}$. This study also reports only 3-month outcomes, which is well below the recommended follow-up time frame. Two of these patients were Risser 1 and 2 at baseline, whereas the other 2 were Risser 4. All should have been followed to Risser 5. These outcomes are summarized in Table 1.

\section{Manipulation Plus Traction}

Two studies reported on results obtained using chiropractic manipulation plus traction methods. One of these was a case report on an 11-year-old female patient with a $22^{\circ}$ left thoracolumbar scoliosis. ${ }^{22}$ The patient was treated using chiropractic manipulation plus positional traction, with the patient lying left recumbent over a foam roll for a total of 31 visits over 3 months. Posttreatment radiography revealed a curve reduction of $3^{\circ}$.

In a retrospective case series, Stitzel et al published the results of patients using a proprietary traction chair for scoliosis treatment. ${ }^{23}$ Results at baseline and 6 months were reported for a total of 15 patients. This paper aimed to examine how apical rotation changes occurring while sitting in the traction chair may affect the curve measurement at 6 months. They found that the Cobb angle was likely to increase in those patients whose apical rotation initially increased during in-chair radiographs or had an initial curve apex above T11. In contrast, their data indicated insignificant Cobb angle decreases in patients whose apical rotation improved in-chair. The data for these 2 studies can be found in Table 1 . 
Neither of these studies followed patients for the minimum required time interval, nor did they achieve a correction $>6^{\circ}$ in Cobb angle measurement.

\section{Manipulation Plus Exercises}

The most commonly reported treatment approach was the use of chiropractic manipulation combined with exercise-based methods. We found a total of 16 studies using some form of a combined approach. The respective outcome data are reported in Table 1.

We located 2 case reports and 1 case series in which the Chiropractic BioPhysics (CBP) technique was used. In the case series, 7 patients aged 6 to 17 years were reported, 4 of whom had an average Cobb angle measurement of $16.2^{\circ} .{ }^{24}$ The average Cobb angle after 3 months of care, including 38 clinical treatments, was $11.6^{\circ}$. This is considered a valid stabilization goal of $\pm 5^{\circ}$ according to the previously outlined criteria. However, because Risser or Sanders staging is not reported, it is difficult to place this result in its proper context, aside from the fact that the results are only after 3 months.

Nektalov described the treatment of a 21-year-old woman also using CBP technique. ${ }^{25}$ The patient was treated and followed for 3 months, undergoing a total of 36 treatments within that period. Although clinical pain scale scores and radiographic improvements in sagittal spine parameters were reported, posttreatment Cobb angle measurements were not reported.

In the third CBP case report we found, Jones described the results for a 14-year-old girl treated 15 times over 13 weeks. ${ }^{26}$ The patient had a baseline Risser 1 and a Cobb angle of $17.2^{\circ}$. Posttreatment radiographs revealed a $13.5^{\circ}$ Cobb angle, with clinical improvements observed in visual posture analysis. Although a baseline SF-36 was completed, the report does not discuss if any follow-up SF-36s were additionally completed.

A case study by Ray et al described the treatment of a 75 -year-old man with a $10^{\circ}$ lumbar scoliosis. ${ }^{27}$ The patient was treated and followed for 2 years using the Network Spinal Analysis method. The authors reported surface electromyography, paraspinal thermography, and radiographic Cobb angle as the chief outcomes. After 2 years, the Cobb angle was completely resolved. It is unknown from reading this paper if the patient had an adolescent onset of this lumbar scoliosis or if this scoliosis would be more accurately described as an adult degenerative scoliosis. This is certainly plausible given that $68 \%$ of adults over the age of 60 are likely to have degenerative lumbar scoliosis. ${ }^{28}$ Nonetheless, the magnitude of these results warrants further investigation. This was the only article we could find using this particular technique.

One case report with one of the longest total follow-up times was that by Bowler and Conlen. ${ }^{29}$ They outlined the multimodal treatment of a 4 -year-old patient with a history of a $25^{\circ}$ juvenile idiopathic scoliosis, craniosynostosis, and an Arnold-Chiari type 1 malformation. The patient was treated and followed over a 6-year period using a combination of therapies, including upper cervical chiropractic manipulation, craniosacral therapy, raindrop therapy, and cranial manipulation. Although cases of juvenile idiopathic scoliosis are often progressive and eventually require surgical intervention, ${ }^{30}$ the Cobb angle in this patient remained unchanged $\left(25^{\circ}\right.$ at baseline compared with $23^{\circ}$ at age 10). However, follow-up should still be continued on this patient through skeletal maturity to assess end-of-growth results.

Chiropractic manipulation has also been combined with Pilates in a report by Blum describing the treatment of a 39-year-old woman with a history of spinal fusion surgery for adolescent idiopathic scoliosis. ${ }^{31}$ The patient received chiropractic manipulation using the sacro-occipital technique for $>1$ year, and was also referred to a Pilates studio where she worked out for $>1$ year as well. The chief outcomes of the report were the decreased amount of chiropractic treatments needed a year later and the patient's ability to pick up her son again. Although pain and functional outcomes are important parts of any nonsurgical treatment plan, they do not provide the complete picture as to whether or not the Cobb angle can be reduced by the given therapy. To be sure, Cobb angle changes are difficult in a postfusion patient such as this one. However, the patient was not followed for the requisite time, as Weiss ${ }^{7}$ suggested.

In 2004, Morningstar et al published a case series using a multimodal therapy in 22 patients aged $15-65 .^{32}$ The average Cobb angle correction was $17^{\circ}$ for the entire cohort. The lowest correction was $8^{\circ}$, or $100 \%$ of the cohort achieved scoliosis correction. However, the follow-up period was only 6 weeks, vastly short of the recommended 5 years for skeletally mature patients. Morningstar and Joy applied this same multimodal treatment to 3 patients with unique scoliosis presentations: 1 patient was status post-Harrington instrumentation, 1 patient had a concomitant Scheuermann's kyphosis, and the third patient had a left thoracic scoliosis. ${ }^{33}$ In all 3 cases, the minimum correction was $8^{\circ}$, and clinical improvements in pain and functional status were achieved for all patients. Again, however, the longest follow-up was only 24 weeks. This is well below the recommended 5 years. Morningstar and Strauchman used the same multimodal treatment in conjunction with manipulation under anesthesia for a 59-year-old woman with adult degenerative scoliosis. ${ }^{34}$ The rehabilitative therapies were applied for 8 weeks after the manipulation under anesthesia. Clinical improvements in pain and disability were achieved. These improvements were maintained at 6 months. In a description of a case lasting 4 weeks, Morningstar et al outlined the use of these same therapies in a 20 -year-old woman with a $35^{\circ}$ right thoracic scoliosis and reported a correction of $15^{\circ} .{ }^{35}$ At 3 
years after treatment, a follow-up radiographic study revealed continued improvement of $18^{\circ} \cdot{ }^{36}$ Another case series by Morningstar looked at the results of a multimodal chiropractic rehabilitation treatment in adult patients. ${ }^{37}$ After a 2-year follow-up, the average Cobb angle correction was about $9^{\circ}$ across 28 total patients. Similarly, a 14-year-old girl with thoracic and lumbar curves of $24^{\circ}$ and $17^{\circ}$, respectively, was treated using the aforementioned multimodal chiropractic rehabilitation treatment. After 4 years of follow-up and continued home care, the patient's curves measured $12^{\circ}$ in the thoracic spine and $4^{\circ}$ in the lumbar spine. ${ }^{38}$

Dovorany et al published a case report on a set of identical twins who both had a thoracolumbar scoliosis. ${ }^{39}$ The patients participated in a short 2-week trial of chiropractic rehabilitation and then continued to perform a set of prescribed exercises. At follow-up 2 years after skeletal maturity, their curves were corrected from $47^{\circ}$ to $26^{\circ}$ (patient A) and $37^{\circ}$ to $22^{\circ}$ (patient B). Both patients had been scheduled for spinal fusion surgery prior to initiation of the described treatment.

In a case-controlled series, Morningstar et al looked at multimodal chiropractic rehabilitation with the added dimension of neurotransmitter supplementation. ${ }^{40}$ Those patients taking the neurotransmitter supplementation achieved better Cobb angle corrections at 6 months compared with those who opted against neurotransmitter treatment.

Finally, one case report described the physical therapy of a 9-year-old patient with a double major scoliosis pattern measuring $18^{\circ}$ and $24^{\circ}$ who was treated and followed for 6 months. At that time, both curves measured $11^{\circ} .^{41}$ The treatment rendered was a combination of spinal manipulation and exercises as administered by a physical therapist. They also reported improvements in the SRS-22 as well as the Bad Sobernheim Stress Questionnaire and Brace Questionnaire.

\section{DisCUSSION}

Despite the case reports and case series cited above, only 2 reported outcomes consistent with consensus criteria. In both studies, ${ }^{41,42}$ skeletally immature patients were followed through skeletal maturity and at least 2 years thereafter. There were no studies that followed adult patients for the recommended 5 years. Many of these studies, regardless of the specific modalities employed, were able to document specific quality-of-life improvements, such as pain. Given that pain is among the chief reasons adults with scoliosis seek treatment, ${ }^{42}$ many of these treatment regimens may hold value even when they cannot produce radiographic curve changes.

Many of the studies are case reports or case series with 2 or 3 cases. In these instances, although the posttreatment
Cobb angle was reported, it would not be appropriate to calculate the percentage of cases who saw improvement, stabilization, or progression. However, it is important that future chiropractic studies seek to align the reported Cobb angle differences in terms of these categories (percentage of cases that improved $>6^{\circ}$, percentage that remained $\pm 5^{\circ}$ from baseline, and those that progressed $>6^{\circ}$ ). For example, Chen and Lantz interpreted a value of $\pm 4^{\circ}$ as unchanged. ${ }^{14} \mathrm{By}$ current criteria this would instead need to be $\pm 5^{\circ}$. In singular case reports, where it is impossible to calculate these percentages, authors should still report their Cobb angle changes. However, they should define these changes within the aforementioned metrics recommended by SOSORT/SRS.

In looking at the data in general, few studies included baseline Risser staging, although some studies stated that these data were, in fact, collected. Given that fact that scoliosis progression differs significantly at various Risser stages, ${ }^{43}$ it is important that future chiropractic studies report the initial Risser stage, as well as the stage at which treatment was discontinued. For example, a Cobb angle that remained unchanged from Risser 0 to Risser 3 may have been more significant than a $6^{\circ}$ curve correction during Risser 4.

The quality of chiropractic research for scoliosis treatment is low. Among all of the studies reviewed here, only one had a randomized clinical trial design ${ }^{20}$; another was a times series cohort. ${ }^{14}$ The remaining studies were case series or case reports. Although they are valuable, they are not enough to produce reliable treatment guidelines within the chiropractic profession. This is likely why the Council on Chiropractic Guidelines and Practice Parameters Guidelines essentially mirror the SOSORT consensus paper for idiopathic scoliosis. Higher-quality studies need to be produced. Additionally, only 2 of the studies reviewed followed patients for the minimum recommended time. Therefore, future chiropractic studies on scoliosis treatment should seek to follow immature patients through Risser 5 and to follow adult patients for a minimum of 5 years after treatment initiation. We encourage any and all future chiropractic authors to use the SOSORT criteria to report their treatment outcomes. This will only serve to elevate the level of quality of scoliosis-related research within the chiropractic profession.

\section{Limitations}

Although unlikely, because the original version of the SOSORT consensus paper was published in 2005, articles on chiropractic scoliosis treatment published prior to January of 2000 may have reported their results consistent with the SOSORT criteria. We also limited our search terms. A more expanded set of key search terms may have yielded more results to review. Finally, this is not a qualitative review. Therefore, this article does not 
speak to the quality or strength of any of the articles reviewed herein. Our main purpose was to evaluate the extent to which currently published chiropractic authors have published their results in a manner recommended by SOSORT.

It is important to note that the 2014 SOSORT and SRS consensus paper was published in 2015, after the vast majority of these reviewed chiropractic studies were published.

\section{CONCLUSION}

The majority of chiropractic studies published after publication of the initial SOSORT/SRS consensus paper for the treatment of idiopathic scoliosis did not report their outcomes as recommended by SOSORT/SRS consensus criteria. These criteria may provide future authors with a template with which to analyze and report future chiropractic treatment data specifically regarding idiopathic scoliosis.

The collective body of chiropractic research related to scoliosis treatment is of low quality by study design. Higher-quality research designs, combined with reporting of outcomes as recommended by SOSORT/SRS criteria, will aid in the future development of chiropractic treatment guidelines for the management of scoliosis.

\section{Funding SOURCES AND CONFlictS of INTEREST}

No funding sources or conflicts of interest were reported for this study.

\section{CONTRIBUTORSHIP INFORMATION}

Concept development (provided idea for the research): M.W.M., C.J.S., A.S., B.D.

Design (planned the methods to generate the results): M.W.M., C.J.S., A.S., B.D.

Supervision (provided oversight, responsible for organization and implementation, writing of the manuscript): M.W.M.

Data collection/processing (responsible for experiments, patient management, organization, or reporting data): M.W.M., C.J.S.

Analysis/interpretation (responsible for statistical analysis, evaluation, and presentation of the results): M.W.M., B.D.

Literature search (performed the literature search): A.S., B.D.

Writing (responsible for writing a substantive part of the manuscript): M.W.M.

Critical review (revised manuscript for intellectual content, this does not relate to spelling and grammar checking): A.S., B.D., C.J.S.

\section{Practical Applications}

- A total of 271 studies have been published on chiropractic treatment of scoliosis since the year 2000.

- The SOSORT criteria should be used to evaluate the impact of given nonsurgical treatment.

- This study identifies the flaws in current chiropractic research regarding scoliosis and provides direction for future study designs and outcome reporting.

\section{REFERENCES}

1. Moen KY, Nachemson AL. Treatment of scoliosis: an historical perspective. Spine (Phila Pa 1976). 1999;24(24): 2570-2575.

2. Hawes MC. The use of exercises in the treatment of scoliosis: an evidence-based critical review of the literature. Pediatr Rehabil. 2003;6(3-4):171-182.

3. Baer HA. Divergence and convergence in two systems of manual medicine: osteopathy and chiropractic in the United States. Med Anthropol Q. 1987;1(2):176-193.

4. Johnson C. Use of the term subluxation in publications during the formative years of the chiropractic profession. J Chiropr Humanit. 2011;18(1):1-9.

5. Feise RJ. An inquiry into chiropractors' intention to treat adolescent idiopathic scoliosis: a telephone survey. J Manip Physiol Ther. 2001;24(3):177-182.

6. Negrini S, Hresko TM, O'Brien JP, et al. Recommendations for research studies on treatment of idiopathic scoliosis: Consensus 2014 between SOSORT and SRS Non-Operative Management Committee. Scoliosis. 2015;10:8.

7. Weiss HR. Physical therapy intervention studies on idiopathic scoliosis: review with the focus on inclusion criteria. Scoliosis. 2012;7(1):4.

8. Weiss HR, Negrini S, Hawes MC, et al. Physical exercises in the treatment of idiopathic scoliosis at risk of brace treatment: SOSORT consensus paper 2005. Scoliosis. 2006;1:6.

9. Council on Chiropractic Guidelines and Practice Parameters (CCGPP). Chiropractic management of thoracic spine conditions. Available at http://www.clinicalcompass.org 2009 Accessed February 21, 2016.

10. Jaszewski E, Sorbara A. Improvement in a child with scoliosis, migraines, attention deficit disorder and vertebral subluxations utilizing the Pierce chiropractic technique. $J$ Pediatr Matern Fam Health Chiropr. 2010;2010(1):30-34.

11. Khauv KB, Dickholtz M. Improvement in adolescent idiopathic scoliosis in a patient undergoing upper cervical chiropractic care: a case report. J Pediatr Matern Fam Health Chiropr. 2010;2010(4):136-142.

12. Chung J, Salminen B. Reduction in scoliosis in a 10 year-old female undergoing upper cervical chiropractic care: a case report. J Pediatr Matern Fam Health Chiropr. 2011;2011(1): 23-30.

13. Chen KC, Chiu EHH. Adolescent idiopathic scoliosis treated by spinal manipulation: a case study. J Altern Complement Med. 2008;14(6):749-751. 
14. Chen J, Lantz CA. Effect of chiropractic intervention on small scoliotic curves in younger subjects: a time-series cohort design. J Manip Physiol Ther. 2001;24(6):385-393.

15. Waldrop R, Ouellette B, Tabick L. Management of adolescent idiopathic scoliosis using the SpineCor brace system: a case study. J Pediatr Matern Fam Health Chiropr. 2011;2011(4): 115-119.

16. Morningstar M. Outcome observations in patients using a scoliosis activity suit: a retrospective chart review after oneyear follow-up. J Scoliosis Rehabil. 2013:1-10.

17. Morningstar MW, Siddiqui A, Stitzel CJ, Dovorany B. Pain and radiographic outcomes in adult idiopathic scoliosis patients using a scoliosis activity suit: An 18-month case controlled chart review. Clin Med. 2015;6(9):597-604.

18. Golembiewski GV, Catanzaro DJ. Scoliosis reduction utilizing an exercise. J Vertebral Subluxation Res. 2001;4(2): 31-36.

19. Lewis C, Erhard R, Drysdale G. Kyphoscoliosis improvement while treating a patient for adhesive capsulitis using the active therapeutic movement version 2. J Manip Physiol Ther. 2008; 31(9):715-722.

20. Rowe DE, Feise RJ, Crowther ER, et al. Chiropractic manipulation in adolescent idiopathic scoliosis: a pilot study. Chiropr Osteopat. 2006;14:15.

21. Morningstar M. Integrative treatment using chiropractic and conventional techniques for adolescent idiopathic scoliosis: outcomes in four patients. J Vertebral Subluxation Res. 2007; 2007(Jul 9):1-7.

22. Wendland-Colby C, Addison DL. Curve improvement in a patient with adolescent idiopathic scoliosis following chiropractic care: a case report. J Pediatr Matern Fam Health Chiropr. 2012;2012(3):77-80.

23. Stitzel CS, Dovorany B, Morningstar MW, Siddiqui A. Clinical evaluation of the ability of a proprietary scoliosis traction chair to de-rotate the spine: 6-month results of Cobb angle and rotational measurements. Clin Pract. 2014;4(2): 50-52.

24. Fedorchuk C, Wetterlin JK, McCoy M. Reduction of subluxation using CBP technique and improvement of childhood idiopathic scoliosis: a series of seven cases. $J$ Pediatr Matern Fam Health Chiropr. 2010;2010(Dec 29): 220-222.

25. Nektalov B. Improvement in a patient with scoliosis undergoing chiropractic care: a case study. J Pediatr Matern Fam Health Chiropr. 2012;2012(Mar 22):31-37.

26. Jones D. Reduction in adolescent idiopathic scoliosis following chiropractic care: a case study. J Pediatr Matern Fam Health Chiropr. 2013;2013(Apr 11):28-33.

27. Ray K, Knowles D, Knowles R. Reduction of a lumbar scoliosis \& improved cervical curve in a geriatric patient following Network Spinal Analysis ${ }^{\mathrm{TM}}$ care: a case study. Ann Vertebral Subluxation Res. 2013;2013(Jun 10):18-28.
28. Schwab F, Dubey A, Gamez L, et al. Adult scoliosis: prevalence, SF-36, and nutritional parameters in an elderly volunteer population. Spine. 2005;30:1082-1085.

29. Bowler B, Conlen K. An interdisciplinary approach to juvenile idiopathic scoliosis, craniosynostosis, and Chiari I malformation. J Pediatr Matern Fam Health Chiropr. 2012; 2012(May 21):54-60.

30. Robinson CM, McMaster MJ. Juvenile idiopathic scoliosis: curve patterns and prognosis in one hundred and nine patients. J Bone Joint Surg Am. 1996;78:1140-1148.

31. Blum CL. Chiropractic and Pilates therapy for the treatment of adult scoliosis. J Manip Physiol Ther. 2002;25(4):E3.

32. Morningstar MW, Woggon D, Lawrence G. Scoliosis treatment using a combination of manipulative and rehabilitative therapy: a retrospective case series. BMC Musculoskelet Disord. 2004;5:32.

33. Morningstar MW, Joy T. Scoliosis treatment using spinal manipulation and the Pettibon Weighting System: a summary of 3 atypical presentations. Chiropr Osteopat. 2006;14:1.

34. Morningstar MW, Strauchman MN. Management of a 59year-old female patient with adult degenerative scoliosis using manipulation under anesthesia. J Chiropr Med. 2010;9(2): 77-83.

35. Morningstar MW, Strauchman MN, Gilmour G. Adolescent idiopathic scoliosis treatment using Pettibon corrective procedures: a case report. J Chiropr Med. 2004;3(3):96-103.

36. Morningstar M, Strauchman M. Adolescent idiopathic scoliosis treatment using the Pettibon corrective procedures: a case report [Letter to the Editor]. J Chiropr Med. 2007;6(2): 83-84.

37. Morningstar MW. Outcomes for adult scoliosis patients receiving chiropractic rehabilitation: a 24-month retrospective analysis. J Chiropr Med. 2011;10(3):179-184.

38. Morningstar M. Four-year follow-up of a patient undergoing chiropractic rehabilitation for adolescent idiopathic scoliosis. J Pediatr Matern Fam Health. 2011;2011(May 16):54-58.

39. Dovorany B, Morningstar MW, Stitzel C, Siddiqui A. Results of chiropractic scoliosis rehabilitation treatment at two years post-skeletal maturity in identical female twins. J Bodyw Mov Ther. 2015;19(4):592-596.

40. Morningstar MW, Siddiqui A, Dovorany B, Stitzel CJ. Can neurotransmitter status affect the results of exercise-based scoliosis treatment? Results of a controlled comparative chart review. Altern Integr Med. 2014;3:4.

41. Villafañe JH, Silva GB, Dughera A. Manipulative and rehabilitative therapy as a treatment of idiopathic scoliosis without psychological sequelae: a case report. J Chiropr Med. 2012;11(2):109-114.

42. Rigo M. Differential diagnosis of back pain in adult scoliosis (non-operated patients). Scoliosis. 2010;5(suppl 1):O44.

43. Reamy BV, Slakey JB. Adolescent idiopathic scoliosis: review and current concepts. Am Fam Physician. 2001;64(1):111-116. 